\title{
Parametrically forced sine-Gordon equation and domain walls dynamics in ferromagnets
}

\author{
Vadim Zharnitsky \\ Division of Applied Mathematics, Brown University, Providence, Rhode Island 02912 \\ Igor Mitkov \\ Applied Theoretical and Computational Physics Division and Center for Nonlinear Studies \\ Los Alamos National Laboratory, Los Alamos, New Mexico 87545 \\ Mark Levi \\ Department of Mathematical Sciences, Rensselaer Polytechnic Institute, Troy, New York 12180
}

(April 30, 2018)

\begin{abstract}
A parametrically forced sine-Gordon equation with a fast periodic mean-zero forcing is considered. It is shown that $\pi$-kinks represent a class of solitary-wave solutions of the equation. This result is applied to quasi-one-dimensional ferromagnets with an easy plane anisotropy, in a rapidly oscillating magnetic field. In this case the $\pi$-kink solution we have introduced corresponds to the uniform "true" domain wall motion, since the magnetization directions on opposite sides of the wall are anti-parallel. In contrast to previous work, no additional anisotropy is required to obtain a true domain wall. Numerical simulations showed good qualitative agreement with the theory.
\end{abstract}

75.60.Ch 03.40.Kf

The sine-Gordon equation (SGE) arises in various physical applications including Josephson junction transmission lines [1],2], dislocations in crystals [3], charge density waves [4], waves in quasi-one-dimensional ferromagnetic materials [5]. The only stable traveling wave solutions of ordinary SGE for a scalar field $\phi$ are localized solutions with identical boundary conditions $\phi=0$ and $\phi=2 \pi$, called $2 \pi$-kinks. On the other hand, nonlocalized kinks separating regions with different values of the field (e.g. $\pi$-kinks) are also important since they are easier to follow experimentally. This is especially relevant for domain wall dynamics in quasi-one-dimensional ferromagnets with strong anisotropy of a hard magnetization axis [0 7,9,10]. In the presence of an external magnetic field (weak compared to the anisotropy) applied within the easy plane, the spin dynamics can be described by SGE (see, e.g., [6]) with $2 \pi$-kinks as the only traveling wave solutions. These localized solutions, preserving the average magnetization, were observed experimentally [11, 12]. An interesting effect of evolution of the average magnetization takes place if adjacent domains have anti-parallel magnetizations which correspond to $\pi$ kinks or "true" domain walls. Such $\pi$-kinks were obtained by introducing additional anisotropy within the easy plane (see, e.g., [6] and [13], sect. 8.3).

In this letter we obtain $\pi$-kinks in a modified SGE with a fast mean-zero parametric excitation. For quasi-onedimensional ferromagnets with an easy plane anisotropy, this implies the existence of true domain walls generated by a rapidly oscillating external magnetic field. As opposed to [6,13], no additional anisotropy of the ferromagnet is required for the existence of $\pi$-kinks.

Various cases of parametrically forced SGE have arisen in numerous models of physical systems [2, 10, 14, 15. However, the mean-zero case has been left out of consideration, while it is significant both for theory and applications. Indeed, a zero-average periodic forcing implies that the states $\phi=0$ and $\phi=\pi$ are symmetric. This leads to the existence of $\pi$-kinks with such time dependent macroscopic quantity as the spatial average of the field.

We start with the Hamiltonian 6,16

$$
\mathcal{H}=\sum_{i=1}^{N}\left[-J \mathbf{S}_{i} \cdot \mathbf{S}_{i+1}+D\left(S_{i}^{z}\right)^{2}-g \mu_{B} H S_{i}^{x}\right],
$$

where $\mathbf{S}_{i}$ are dimensionless classical spin vectors, $N$ is the number of spins, and $J$ is a constant of exchange interaction. The external magnetic field $\mathbf{H}$ is directed along the $X$-axis. The anisotropy constant $D$ provides the existence of an easy plane, $X Y$, at each site of the chain.

The dynamics of a spin $\mathbf{S}_{i}$ in the effective magnetic field is governed by 13

$$
\hbar \dot{\mathbf{S}}_{i}=-\mathbf{S}_{i} \times \frac{\partial \mathcal{H}}{\partial \mathbf{S}_{i}} .
$$

Representing spins in spherical coordinates, $\mathbf{S}_{i}=$ $S\left(\cos \theta_{i} \cos \phi_{i}, \cos \theta_{i} \sin \phi_{i}, \sin \theta_{i}\right)$, and taking the continuum limit, yields a system of two first-order (in time) partial differential equations for $\theta$ and $\phi$ [6. 16]. When the condition $D S \gg g \mu_{B} H$ holds [6], the system reduces to SGE for the polar angle in the easy plane, $\phi$

$$
\phi_{x x}-\frac{\ddot{\phi}}{C^{2}}=\nu H \sin \phi
$$


where $C=2 a_{0} S^{2} \sqrt{J D} / \hbar, \nu=g \mu_{B} / 2 J S a_{0}^{2}$, and $a_{0}$ is the lattice constant. We take the external field $H=$ $H_{0} a(t / \epsilon)$, where $a$ is a mean-zero periodic function with a unit amplitude, and $H_{0}$ is the amplitude of the field. Rescaling time and coordinate to dimensionless variables, $\tilde{t}=t C \sqrt{\nu H_{0}}, \tilde{x}=x \sqrt{\nu H_{0}}$, we obtain (after dropping tildes) a parametrically forced SGE (PSGE)

$$
\phi_{t t}-\phi_{x x}+a(t / \epsilon) \sin \phi=0 .
$$

After averaging Eq. (1) directly, over the fast time scale $\epsilon$, we are left with a linear wave equation with wave propagation velocity $c= \pm 1$. In what follows, we use more subtle averaging to obtain $\pi$-kink solutions moving with any prescribed velocity.

The phenomenon of $\pi$-kinks in PSGE has a finitedimensional counterpart: it is the stabilization of the inverted pendulum by periodic vibration of its suspension point (Kapitza pendulum [17]). Since this latter phenomenon is responsible for the existence of $\pi$-kinks, we outline a very simple geometrical explanation of stability of the inverted pendulum with a vibrating suspension. Full details with other applications can be found in 20] along with further references to numerous papers on the subject. Consider a pendulum (a bob on a massless rod of length $l$ ) in a nearly upside-down position, with the suspension point undergoing vertical vibration. We assume the latter to have high acceleration and small amplitude. Since the acceleration is large, the force of the rod on the bob is large so that the bob would be expected to follow, in the first approximation, the direction of the rod. This suggests considering an auxiliary system where the velocity of the bob is actually constrained to the line of the rod. In this case the bob will oscillate along an arc of a tractrix (the "pursuit" curve: all tangent segments from this curve to a straight line have the same length) and thus will be a subject to an average centrifugal force $m k\left\langle v^{2}\right\rangle$, where $k$ is the curvature of the tractrix and $v$ is the speed of the bob which is approximately the speed of the suspension point when the pendulum is near the top. If we now release the constraint, thus releasing the centrifugal force, the bob will behave as if it were subject to a centripetal force $m k\left\langle v^{2}\right\rangle$ which pushes the pendulum towards the top. If this force exceeds the gravitational force, the pendulum is stable; this leads to the simple stability criterion $\left\langle v^{2}\right\rangle>\ell g$, see [20].

To average Eq. (4) we apply a series of canonical nearidentical transformations via the normal form technique 18, 19, so as to bring the original equation to a better form with rapidly oscillating coefficients moved to higher order terms. Since the transformations are near-identical the solutions for the reduced Hamiltonian are close to those for the original one.

The Hamiltonian of PSGE (4) is given by

$$
H=\int_{-\infty}^{+\infty}\left(\frac{p^{2}}{2}+\frac{\phi_{x}^{2}}{2}-a \cos \phi\right) d x
$$

where $p \equiv \phi_{t}$ (below we omit the limits of integration and $d x$ ). Let the first canonical transformation be defined implicitly as follows

$$
p=p_{1}+\frac{\delta W_{1}\left(\phi, p_{1}\right)}{\delta \phi}, \quad \phi_{1}=\phi+\frac{\delta W_{1}\left(\phi, p_{1}\right)}{\delta p_{1}} .
$$

The new Hamiltonian is given by $H_{1}=H+W_{1 t}$, or

$$
H_{1}=\int\left[\frac{1}{2}\left(p_{1}+\frac{\delta W_{1}}{\delta \phi}\right)^{2}+\frac{\phi_{x}^{2}}{2}-a \cos \phi\right]+W_{1 t} .
$$

To kill the rapidly oscillating term in $H$ we choose $W_{1}=$ $\epsilon \int a_{-1} \cos \phi$, where $a_{-1}$ is an anti-derivative with zero average. The Hamiltonian takes the form

$$
H_{1}=\int \frac{p_{1}^{2}}{2}+\frac{\phi_{1 x}^{2}}{2}-\epsilon a_{-1} p_{2} \sin \phi_{1}+\frac{1}{2} \epsilon^{2} a_{-1}^{2} \sin ^{2} \phi_{1} .
$$

The last term in the above Hamiltonian cannot be removed by near-identity transformations since it has nonzero mean with respect to $t$. However, all other terms with rapidly oscillating coefficients can be killed. Thus, choosing $W_{2}=\epsilon^{2} \int a_{-2} p_{2} \sin \phi_{1}$ we obtain the Hamiltonian $H_{2}=\int \frac{p_{2}^{2}}{2}+\frac{\phi_{2 x}^{2}}{2}+\frac{1}{2} \epsilon^{2}\left\langle a_{-1}^{2}\right\rangle \sin ^{2} \phi_{2}+\epsilon^{2} R+O\left(\epsilon^{2}\right)$, where $\langle R\rangle=0$. Finally, taking $W_{3}=\epsilon^{3} \int R_{-1}$ we obtain the Hamiltonian

$$
H_{3}=\int\left(\frac{p_{3}^{2}}{2}+\frac{\phi_{3 x}^{2}}{2}+\frac{1}{2} \epsilon^{2}\left\langle a_{-1}^{2}\right\rangle \sin ^{2} \phi_{3}\right)+O\left(\epsilon^{3}\right) .
$$

After rescaling $X=\epsilon x, T=\epsilon t, P=2 \epsilon^{-1} p_{3}, \Phi=2 \phi_{3}$ in the equations of motion corresponding to (9), we obtain

$$
\left\{\begin{array}{l}
\Phi_{T}=P+O\left(\epsilon^{2}\right) \\
P_{T}=\Phi_{X X}-\left\langle a_{-1}^{2}\right\rangle \sin \Phi+O(\epsilon) .
\end{array}\right.
$$

The system (10) is a slightly perturbed SGE with $2 \pi$ kinks as approximate solutions. After rescaling back to variables $\left(\phi_{3}, p_{3}\right)$, we obtain $\pi$-kinks as approximate solutions $\phi_{3} \approx U(x, t)$, where

$$
U(x, t)=2 \arctan \left[\exp \left(\epsilon \sqrt{\left\langle a_{-1}^{2}\right\rangle} \frac{x-c t}{\sqrt{1-c^{2}}}\right)\right] .
$$

Note, that by using the normal form technique, our equation (4) has been brought to the form in which it explicitly represents a slightly perturbed SGE (10). Since we have employed only near-identical transformations, the original equation (4) should have solutions close to the solitary waves given by Eq. (11).

We have verified our results by the numerical simulations of PSGE (1), using the second-order leap-frog method. To obtain initial conditions for the original variables, $\phi(x, 0), p(x, 0)$, we start with the initial conditions for the transformed variables, $\phi_{3}, p_{3}$, generated from Eq. (11) as $\phi_{3}(x, 0)=U(x, 0), p_{3}(x, 0)=U_{t}(x, 0)$. Retracing our canonical transformations and keeping the lowest order terms only we arrive at 


$$
\begin{aligned}
& \phi(x, 0)=U(x, 0), \\
& p(x, 0)=U_{t}(x, 0)-\epsilon a_{-1}(0) \sin U(x, 0) .
\end{aligned}
$$

In Figure 11 we compare the results of the simulations (dashed line) with the analytical solution (11) (thin solid line). One can see from the Figure a good quantitative agreement between the theory and numerical simulations (the two curves in the Figure are almost indistinguishable). We have also simulated PSGE (4), starting with initial conditions of shapes different from (12). We have found that these solutions split into two linear wave packets moving in opposite directions with velocities $c= \pm 1$. This can be explained by the closeness of PSGE with mean-zero excitation to the linear wave equation, as was mentioned above.

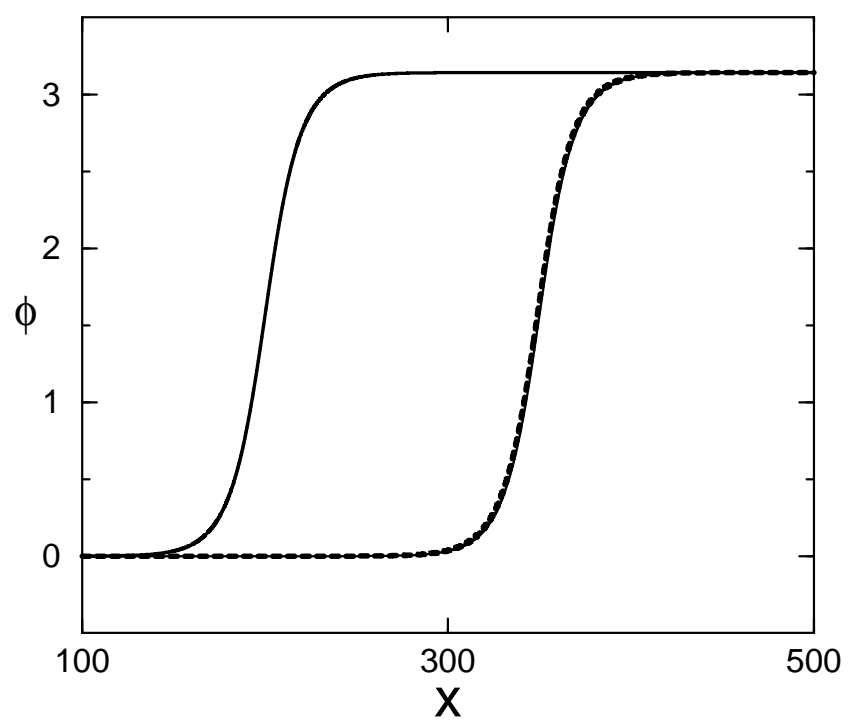

FIG. 1. The behavior of $\pi$-kink solution in the PSGE (4). The thick solid line corresponds to the initial profile given by (12) with $c=1 / 2, a=\sin (t / \epsilon)$. The thin solid and dashed lines correspond to the approximate solution (11) and the result of simulation, respectively, at $t=300$. The analytical and numerical curves almost coincide. The parameters of the simulations are $\epsilon=0.1$, time step $d t=0.01$, mesh size $d x=0.05$, system size $L=500$.

In terms of the original physical system described by Eq. (3), the $\pi$-kink solutions represent moving true domain walls generated by a rapidly oscillating external magnetic field. Now we return from the dimensionless variables to the original variables $t=\tilde{t} / C \sqrt{\nu H_{0}}, x=$ $\tilde{x} / \sqrt{\nu H_{0}}$, to estimate the values of physical parameters, which provide the formation of a realistic domain wall. The lattice constant $a_{0}$ and energy constants $J$ and $D$ are taken, according to Ref. 11, as $a_{0} \sim 5 \AA$, and $J \sim D \sim 10 \mathrm{~K}$. In dimensionless units the frequency $\tilde{\omega}$ of the field and the width of the domain wall $\Delta \tilde{x}$ are of order $\epsilon^{-1}$. Then, for the field amplitude $H_{0} \sim 1 G$ and $\epsilon \sim 0.1 \div 0.01$, we obtain $\omega \sim 10^{9} \mathrm{~Hz}, \Delta x \sim 10 \mu \mathrm{m}$. Magnetic field $H_{0} \sin \omega t$, with such values of $H_{0}$ and $\omega$ can be created in an experiment, to observe the predicted effect.

In summary, we have found $\pi$-kink solutions to PSGE with a fast periodic mean-zero forcing. As applied to quasi-one-dimensional ferromagnets with the only anisotropy of an easy plane, these solutions imply moving true domain walls, generated by a rapidly oscillating magnetic field. Our theoretical results are in a good quantitative agreement with numerical simulations of the PSGE. These results are also applicable to essentially two- and three-dimensional easy plane ferromagnets, when plane front solutions are stable with respect to small deformations. This problem, as well as the stability of $\pi$-kinks in PSGE, will be addressed in future investigations.

We would like to thank S. Brazovsky, R. Camassa and $\mathrm{V}$. Beilin for fruitful discussions.

[1] D. W. McLaughlin and A. C. Scott, Physical Review A 18, 1652 (1978).

[2] M. B. Mineev and V. V. Shmidt, Sov. Phys. JETP 52, 453 (1980).

[3] F. C. Frank and J. H. van der Merwe, Proc. R. Soc. London, Ser. A 198, 205 (1949).

[4] M. Rice, A. R. Bishop, J. A. Krumhansl, and S. E. Trullinger, Phys. Rev. Lett. 36, 432 (1976).

[5] K. M. Leung et al., Phys. Rev. B 21, 4017 (1980).

[6] K. M. Leung, Phys. Rev. B 27, 4017 (1983).

[7] H. J. Mikeska, J. Phys. C 11, L29 (1978).

[8] A. R. Bishop and T. F. Lewis, J. Phys. C 12, 3811 (1979).

[9] F. H. Abdullaev and P. K. Habibullaev, Dynamics of solitons in non-homogeneous condensed media (Tashkent, FAN, 1986) (in Russian)

[10] V. M. Yeleonosky and N. N. Kirova, Zh. Eksp. Teor. Fiz. 75, 2210 (1978).

[11] J. K. Kijems, M. Steiner, Phys. Rev. Lett. 41, 1137 (1978); M. Steiner, Z. Phys. B 53, 117 (1983).

[12] L. J. De Jough, Phys. Rev. Lett. 471672 (1981).

[13] A. M. Kosevich, B. A. Ivanov, and A. S. Kovalev, Phys. Rep. 194, 117 (1990).

[14] Yu. S. Kivshar, N. Gronbech-Jensen, and R. D. Parmentier, Phys. Rev. E 49, 4542 (1994).

[15] Yu. S. Kivshar, N. Gronbech-Jensen, and M. R. Samuelsen, Phys. Rev. B 45, 7789 (1992).

[16] J. Wysin, A. R. Bishop, and P. Kumar, J. Phys. C 17, 5975 (1984).

[17] L.D. Landau, M. Lifshitz, Mechanics, (Pergamon Press, Oxford 1960).

[18] V. I. Arnold, Geometrical methods in the theory of ordinary differential equations, (Springer-Verlag 1983).

[19] A. I. Neistadt, J. Appl. Math. Mech. 45(1981), no. 1, 58-63(1982).

[20] M. Levi, Curvature effects in averaging with applications, preprint (1997). 